\title{
Evidence-based medicine: ethically obligatory or ethically suspect?
}

In the pages of Evidence-Based Mental Health and elsewhere, authors have argued that it is ethically obligatory to practice evidence-based medicine (EBM). ${ }^{1-3}$ The argument in favour of this position begins with the assumption that EBM is more able than other strategies to provide us with accurate information about the effectiveness of medical interventions. Accurate information is essential to making clinical recommendations that will be effective in optimising our patients' health. Optimising patients' health is a basic ethical duty for medical practitioners. Therefore, the most accurate information-delivered by EBM-is required for practitioners to fulfil a primary ethical obligation. Similarly, knowingly using less accurate information is less likely to optimise patients' health and is therefore inherently unethical.

This ethical argument relies on an epistemological assumption-that EBM provides us with a more reliable way of knowing than pre-EBM medicine. To date, there is no body of evidence, although there are case examples, demonstrating that EBM is more able to generate accurate medical information than pre-EBM medicine. Furthermore, it is also uncertain whether EBM achieves its ultimate goal of improving patient health compared with previous modes of practice. In the absence of such evidence, EBM relies on this ethical argument to support-and indeed demand-its use.

Because EBM's ethical rationale depends upon the correctness of its epistemological assumption, the assumption must be capable of withstanding scrutiny before one is ethically obliged to practice EBM. In EBM, as the name suggests, it is evidence upon which medical practice is based and it is evidence that will improve the accuracy and reliability of our knowledge. Thus, in order to test EBM's epistemological assumption, it is essential to understand how EBM defines "evidence".

In the authoritative accounts of EBM, "evidence" is never formally defined, but is implied to be quantitative data obtained from research studies-preferably randomised controlled trials or meta-analyses of these trials. Data generated by other research methods are less preferred or located lower down on the "evidence hierarchy". Some consideration is given to non-quantitative research data, such as qualitative data, although the status and weight of such data remains unclear. EBM's proponents also allege EBM's increased reliability by portraying it as inherently neutral, a corrective measure against the biases inherent in any other form of reasoning in clinical practice. There is no mention of the social, political, and economic contexts in which EBM is practiced or any acknowledgement of how these contexts could influence the generation or dissemination of research data. A few examples suggest that this view of EBM as neutral may be unfounded.

"Source of funding bias" is apparent in psychiatric research when we consider the far more rapidly growing body of research on pharmaceuticals compared with psychosocial interventions. Large, private corporations with a commercial interest in pharmaceuticals are a source of funding for researching one type of intervention-medications- whereas there is no equivalent body to favour the funding of other types of psychiatric interventions such as psychosocial treatments. While fewer data do not mean that psychosocial interventions are ineffective, over time the gradual accumulation of research data concerning pharmaceuticals suggests greater evidence of their effectiveness compared with other types of interventions. The greater availability of research data concerning medications as compared to psychosocial interventions is also fostered by "technical bias", which is actually built into EBM's structure. Technical bias favours research that we already know how to do. The ethos of technical bias is aptly captured in step one of EBM's five steps of EBM practice: converting the need for information into an "answerable" question. ${ }^{4}$ As Miettinen points out, there may well be intellectual and clinical value in considering unanswerable questions, or at least, difficult to answer questions. ${ }^{5}$ Furthermore, there may be a gap between what one needs to know, and the answers that the medical research literature can provide. Because EBM prefers certain research methods and certain types of data in its evidence hierarchy, EBM favours those interventions that can best be studied using EBM rules. ${ }^{6}$ For example, research studies of pharmaceuticals are more likely to be conducted according to EBM preferred methods and these methods will generate data that is ranked more highly by EBM (quantitative data from RCTs). Psychotherapy research, on the other hand, is difficult to conduct according to EBM rules-it can never even meet the basic EBM requirement of double blinding. Psychotherapy research methods are also fraught with methodological problems, particularly when compared with studies of pharmaceutical agents. As a result, psychotherapy research data will necessarily be considered tentative and less definitive than data generated by RCTs and meta-analyses of pharmaceuticals.

Source of funding bias and technical bias affect the generation of research data. Publication bias, in which certain types of data are knowingly kept from publication, distorts the dissemination of research data. ${ }^{7}$ Because of publication bias, the total pool of research data from which we draw conclusions about medical interventions cannot be relied upon because it is incomplete. The recent controversy over the use of SSRIs in childhood major depression demonstrates this bias. ${ }^{8}$ In this case, clinical trials which demonstrated no advantage of the active drugs as compared with placebo were not published and not released for professional scrutiny. Conclusions drawn about these drugs by the psychiatric community were made without being aware of these unpublished data.

In light of these examples, it is reasonable to conclude that EBM is not bias free. The social context in which EBM operates cannot be ignored, for this context influences the production of data. But in addition to ignoring potential sources of bias in producing data, EBM also promulgates a view that data alone will tell us which interventions are or are not effective. This view obscures the process by which data becomes evidence. Data do not support conclusions by themselves-they must first be interpreted. Interpretation is 
a process in which judgement is used to evaluate the relevance and weight of data. It is through this process that data are thought to support certain conclusions. However, interpretation is a human process that is necessarily influenced by a variety of factors including power relations, and commercial and other vested interests. ${ }^{9}$ The debate concerning SSRI use in childhood depression again illustrates this point. Now that some leading experts have had the opportunity to examine what we think is the total pool of data on this question, various interpretations of the data have arisen. Garland claims that the total data do not support the view that SSRIs are effective in childhood depression whereas Korenblum disagrees. ${ }^{10}$ This dispute highlights the fact that the same data may be interpreted differently depending on who is examining them, and what knowledge and background they bring to this exercise. Interpreters' judgement may also be affected, consciously or unconsciously, by other factors such as economic concerns, peer pressure, and personal issues of ego and status.

It is important to note that biases and interpretation are not unique to EBM. All knowledge is affected, to different degrees, by these types of factors. However, EBM ignores these issues. This means that the assumption upon which EBM rests-that it is more likely to yield accurate information than any other method-cannot be taken for granted. Indeed, we have good reasons to believe that EBM has significant potential to produce inaccurate information. Because the assumption of greater accuracy does not necessarily hold, then the ethical obligation to practice EBM is also thrown into question.

Even if EBM has these limitations, might it be relatively less inaccurate than pre-EBM medicine? I do not believe it is possible to draw such a conclusion at present given that few of the health interventions that have had the greatest impact on morbidity and mortality have been derived from EBM. Measures such as clean water, handwashing, vaccination, and prenatal care have dramatically improved the health of people. Most of these interventions have arisen through both historical accident and a variety of intellectual developments, including those most derided by EBM, such as pathophysiological reasoning and clinical observation. EBM's ability to optimise patients' health remains to be seen. In the absence of epistemological justification, there is no ethical obligation to practice EBM.

Perhaps the best route to epistemological and ethical justification for EBM will begin with the recognition that all knowledge is tentative and subject to various distortions and error, including knowledge generated by EBM preferred methods. Eliminating distortion is probably impossible. This does not necessarily lead to a nihilistic conclusion that the pursuit of knowledge is pointless. Rather it suggests that what is needed in medical practice is an openness to a plurality of sources of knowledge. Employing the standards of transparency and explicitness championed by EBM, each piece of knowledge can be evaluated on its own terms rather than in accordance to a rigid set of rules which may not be applicable. In this way, we can acknowledge the legitimate challenge posed by EBM - that is, to critically examine the basis of our clinical decisions while at the same remaining open to the diverse means through which knowledge evolves.

MONA GUPTA, MD CM

Department of Psychiatry and Behavioural Neurosciences, McMaster University, Ontario, Canada; mona.gupta@utoronto.ca

\section{REFERENCES}

1 Szatmari P. The art of evidence-based child psychiatry. Evidence-Based Mental Health 2003;6:1-2.

2 Paris J. Canadian psychiatry across 5 decades: from clinical inference to evidence-based practice. Can J Psychiatry 2000;45:34-8.

3 Goldner EM, Abbass A, Leverette JS, et al. Evidence-based psychiatric practice: implications for education and continuing professional development. Can J Psychiatry 2001;46:15 pages following page 424

4 Sackett DL, Straus SE, Richardson WS, et al. Evidence-based medicine: how to practice and teach EBM, 2nd edition. Edinburgh: Churchill Livingstone, 2000.

5 Miettinen O. Commentary on 'A critical appraisal of evidence-based medicine: some ethical considerations'. J Eval Clin Practice 2003:9:123-7.

6 Culpepper L, Gilbert TT. Evidence and ethics. The Lancet 1999;353:829-31.

7 Gilbody SM, Song F. Publication bias and integrity in psychiatric research. Psychol Med 2000;30:253-8.

8 Garland EJ. Facing the evidence: antidepressant treatment in children and adolescents. CMAJ 2004;170:489-91.

9 Leeder SR, Rychetnik L. Ethics and evidence-based medicine. Med J Australia 2001;175:161-4.

10 Mickleburgh R. Antidepressants found ineffective on teenagers. Globe and Mail, 17 February 2004.

\section{Response to Dr Gupta}

t is not uncommon for proponents of evidence-based practice (EBP) to state that once a treatment has been found to be ineffective in a randomised control trial it is "unethical" to continue to practice it. Dr Gupta challenges this statement. She feels there is no justification for stating "we should practice evidence-based mental health because it is ethical". She buttresses her argument by stating that since there are so many concerns about the "truth" of the evidence provided in scientific studies that one should not base the practice of EBP on that foundation.

It is an interesting point but I think it may be dangerous to caricature EBM. I do not recollect any statement by an advocate of EBP saying that the evidence in a randomised controlled trial is the same as "truth". Indeed, I think that what we learn by practicing EBP is rather "the error of our ways". I would admit however, that many advocates of EBP would agree that as the evidence accumulates and insofar as it is consistent (as demonstrated in a meta-analysis), empirical evidence becomes a closer and closer approximation to the truth. It is the "best available evidence", but it will never be the truth itself. Nobody who practices in this way should believe that the truth is a categorical phenomena; that is, true or untrue. Rather, it is "more" or "less" true.

Dr Gupta challenges us not to practice EB mental health because it is ethical. I would ask then, on what basis do I make a clinical decision? If I see a young child with autism, I have a choice: I can refer him to behaviour therapy or not. I would prefer to make my clinical decision on the basis of the best available evidence. What is the alternative to using evidence as a guide? I could do nothing, but that would be clinical paralysis and that surely is unethical. I could make a decision at random by simply flipping a coin, but that does not feel like an ethical thing to do if there is existing evidence (although randomisation in an $\mathrm{N}$ of 1 trial may be the best solution if there is inadequate evidence). I could make my decision on the basis on how I was trained. But as I was 
trained many years ago, that information is now quite out of date. I could instead make my decision based on what I know about the pathophysiology of autism (just like many physicians make clinical decisions about prescribing medication for depression on the basis of their knowledge of neurotransmitters in mood disorders). But as what we know about pathophysiology of autism is so limited (and, I would argue, is equally limited for all psychiatric disorders), I think it is very difficult, if not impossible, to make clinical decisions about treatment based on our very incomplete knowledge of aetiology. Finally, I could make my clinical decision based on my values. What values do I hold about the most appropriate, the most humane, the most empowering form of treatment? In fact, I personally do not find applied behavioural analysis (ABA) very humane. The use of massed discreet trials to teach simple tasks such as matching often looks intrusive and critical. I would much prefer a more developmental sociocognitive approach and indeed such treatments are available but they do not yet have the evidence to support them. But if I were to choose a treatment based on my values over the evidence, I would have to do so by the rules of informed consent. To be ethical, I would have to make that preference known to the parents of this child with autism and they would also have to choose (or not to choose) my values over the evidence. I wonder what a reasonable adult would do in such a circumstance? I bet they would choose treatment that is supported by the evidence over and above my own values and so would choose behaviour therapy.

Do we have an alternative? Instead of saying that it is ethical to practice EBM because the evidence leads one to the "truth", one could say "it is useful to practice EBM". More people tend to get better when a treatment has been shown to be effective by a randomised controlled trial than when an alternative is employed. In this circumstance, more people get better when they receive the "experimental" treatment than when they receive, say, a placebo or the standard treatment. It is not so much a matter of "truthfulness" as it is of "usefulness". Whether the results from a particular study are generalisable to my clinical case load is an open question. But choosing a treatment based on its ability to do good for the most people is a utilitarian approach to the truth, not an absolute categorical glimpse of the truth. It is moreover a value laden statement. EBP is ethical because it produces the most good for the largest number of people (whether or not it is also based on any knowledge of the truth). Surely that is a useful way to treat those with severe mental illness.

PETER SZATMARI, MD Editor, EBMH

\section{Editorial}

\section{The worm turns: publication bias and trial registers revisited}

$\mathrm{R}$ eaders of Evidence-Based Mental Health will be familiar with the fundamental rationale for systematic reviews: reviews should have adequate and well described methods and, in particular, avoid the biased selection of primary studies dependent on their results. A comprehensive and reproducible literature search is now recognised to be a key feature of systematic reviews. We know a lot about the advantages and limitations of electronic bibliographic databases, such as Medline. ${ }^{1}$ There is now a substantial body of empirical evidence about the various kinds of bias that can plague the identification and selection of studies for reviews, including publication bias (studies with significant results are more likely to get published than studies without significant results), English language bias (studies with significant results more likely to be published in English language journals), citation bias (studies with significant results more likely to be cited) and so on. ${ }^{2}$ As long as studies get published somewhere in journals that are indexed in one database or another, then they are potentially retrievable with an adequate search. Publication bias, however, remains the most devastating potential bias. The reasons for the nonpublication of studies vary. The authors of a small study with unexciting results may have little interest or motivation in getting the study published. More pernicious is the situation where a substantial study is not published-and even purposefully suppressed-because the authors or funders do not like the look of the results.

Publication bias has been a perennial concern of reviewers and evidence-based practitioners, but a recent series of events has provided a disturbing example of the potentially serious effects of the failure to publish trial data or to make them available. It has emerged that trials of paroxetine, a selective serotonin reuptake inhibitor, showing negative or neutral results in the treatment of depressive disorders in children and adolescents have been not been published by the trials' sponsor, GlaxoSmithKline. The resulting distortions introduced into systematic reviews are illustrated in this issue of $E B M H$ in the study by Whittington et al [see page 115]. This analysis shows clearly that the inclusion of unpublished data very much reduces the apparent efficacy and increases estimates of the harm of certain medications, in particular paroxetine.

The central problem of how to encourage pharmaceutical companies to become more open about their data has exercised the evidence-based community for a number of years. Companies are clearly concerned about the effects of negative trials on sales of a drug in their drive to recoup the development costs and generate a profit for their shareholders. For this reason, voluntary initiatives to get negative results into the public domain have largely been unsuccessful. In 1998, a clinical trial amnesty was announced. ${ }^{3}$ Despite some initial support from companies (including the then GlaxoWellcome), the amnesty has largely failed-as companies seem to have decided that openness about their products might damage their commercial prospects. It has long been acknowledged that the only satisfactory way of protecting against publication bias is by prospective registration of trials, but such registers remain unsatisfactory. ${ }^{4}$ 
It is hardly surprising, therefore, that the current SSRI case has been seized upon by the general media, medical commentators, and regulatory bodies alike. Here is a great chance to put real pressure on the pharmaceutical companies to adopt a new policy of openness. On 2 June 2004, GlaxoSmithKline was sued by the Attorney General of New York for fraud over the suppression of trial data of importance in the estimation of the efficacy and the potential increase in suicidal risk of paroxetine in children and adolescents. The action was settled and, belatedly, the company has made full trial reports of the unpublished paroxetine trials available on its website (http://www. gsk.com/media/paroxetine.htm). The International Committee of Medical Journal Editors has recently announced that it will no longer accept trials that have not been entered onto a clinical trial database at inception. ${ }^{5}$ Sir Iain Chalmers, meanwhile, has argued that only legislation can force drug companies to make all trial results available. ${ }^{\circ}$

From the point of view of the evidence-based practitioner, we are usually realistic enough in our expectations of our treatments to know that no drug provides benefits without costs. What we need, though, is reliable information about both benefits and costs to give our patients proper information about what they can expect from our recommended therapies. Evidence-Based Mental Health has abstracted two published trials that compared SSRIs with placebo in the treatment of childhood depression ${ }^{78}$ and a summary of the section on the treatment of childhood depression from Clinical Evidence. ${ }^{9}$ Although we believe that the coverage we have given the issue has been true to the status of the published evidence, we are of course unable to report evidence that is not published or is deliberately suppressed. In our view, publication bias subverts evidence-based practice and can harm patients.

We at Evidence-Based Mental Health therefore join the calls on our partners in the pharmaceutical industry to be more transparent and open about their trial data. Failing to do so means, at best, that ineffective treatments are widely used in patients and, at worse, can lead to unnecessary illness and even death if the reported risks of harms are underestimated. We believe that the industry will want to avoid the possibility of increased legislation and cannot afford repeated public relations disasters of this kind.

Lastly, while it is clearly important to make the most of the SSRI case to push for a general improvement in the accessibility of trial results, it is a tragedy that this has involved a treatment for a mental illness. We and our patients are continually fighting stigma, and public perceptions of antidepressants are likely to have become even more negative as a result of this episode.

\section{JOHN GEDDES, MD, PETER SZATMARI, MD, DAVID STREINER, PHD Editors, EBMH}

\section{REFERENCES}

1 Dickersin K, Scherer R, Lefebvre C. Identifying relevant studies for systematic reviews [see comments]. BMJ 1994;309:1286-91.

2 Egger $M$, Smith GD. Bias in location and selection of studies. BMJ 1998;316:61-6.

3 Smith R, Roberts R. An amnesty for unpublished trials. BMJ 1997:315:622.

4 Rennie $D$. Trial registration: a great idea switches from ignored to irresistible. JAMA 2004

5 De Angelis C, Drazen JM, Frizelle FA, et al. Clinical trial registration: a statement from the International Committee of Medical Journal Editors. Lancet 2004;364:911-12.

6 Chalmers I. In the dark: drug companies should be forced to publish all the results of clinical trials. New Sci 2004;181:19.

7 Fleming J. Fluoxetine decreased depressive symptoms in children and adolescents with non-psychotic major depressive disorder. Evid Based Ment Health 1998;1:49.

$8 \mathrm{McCl}$ ure EB, Leibenluff E, Pine DS. Sertraline improves symptoms in children and adolescents with major depressive disorder. Evid Based Ment Health 2004;7:10.

9 Hazell P. Depression in children and adolescents. Evid Based Ment Health 2003;6: 103-4. 